\title{
Splenic Abscess: An Unusual Complication of Colon Cancer
}

\author{
Rishitha Yelisettia, b, Madiha Khurshid ${ }^{\mathrm{a}}$, Areig Awad ${ }^{\mathrm{a}}$, \\ Anand Kaji ${ }^{\mathrm{a}}$
}

\begin{abstract}
Colon cancer is among the common malignancies. Common cancerrelated complications are regional tumor extension, obstruction, and tumor perforation. Abscess formation is among the rare complications. We report a case of a 60 -year-old female who presented with shortness of breath and left upper quadrant pain and was diagnosed with splenic abscess associated with colon cancer. This type of presentation necessitates an early surgical intervention.
\end{abstract}

Keywords: Colon cancer; Splenic abscess

\section{Introduction}

Colorectal cancers are among the most common types of malignancies. Typical presenting features of colorectal carcinomas include a change in the bowel habits with increasing constipation and spurious diarrhea, lower abdominal pain, pencil shaped stools, visible blood in the stool, generalized weakness and weight loss. However, colorectal cancers do not always present with such familiar symptoms. It is a great masquerader and the variability of the presentation is well-documented. Among the less common manifestations of this malignancy include perforation and abscess formation, bacteremia with Streptococcus bovis, Clostridium difficile and extra-intestinal manifestations caused by organisms such as Bacteroides fragilis. Such cases are difficult to diagnose and require unique management strategies. Herein we present one such case of colorectal cancer associated with an abscess and discuss its management from our experience.

\section{Case Report}

We present a case of a 60 -year-old female with no relevant

Manuscript submitted April 21, 2017, accepted May 23, 2017

${ }^{a}$ Department of Internal Medicine, Saint Francis Medical Center, Seton Hall University, Trenton, NJ, USA

${ }^{b}$ Corresponding Author: Rishitha Yelisetti, Department of Internal Medicine, Saint Francis Medical Center, Seton Hall University, Trenton, NJ, USA. Email: ryelisetti@stfrancismedical.org

doi: https://doi.org/10.14740/gr840w past medical history who initially presented to our emergency department with complaint of sudden onset of shortness of breath for 1 day. Patient stated that she woke up from her sleep gasping for air. She denied chest pain, palpitations, wheezing, or cough. Her symptoms were getting progressively worse, prompting a visit to the emergency department. Patient also complained of dull left flank pain, 5/10 in intensity, radiating to the suprapubic region, aggravated by movement and deep inspiration, and relieved partially with analgesics. The pain was not associated with nausea, vomiting, change in her bowel habits, dysuria, urinary urgency or frequency. Her past surgical history was significant for a cesarean section. Family history was remarkable for diabetes and hypertension in mother and breast cancer in sister. She denied active smoking history and no illicit drug use but admitted to consuming 3 - 4 drinks of alcohol once a week. She denied taking any prescription or over the counter medications at home. Review of systems was significant for shortness of breath and minimal fatigue. She denied any changes in her weight or appetite.

On physical examination, her vital signs at the time of presentation were: temperature of $97.8{ }^{\circ} \mathrm{F}\left(36.5^{\circ} \mathrm{C}\right)$, respiratory rate of 18 breaths/min, pulse rate of 122 beats per minute and blood pressure of $89 / 69 \mathrm{~mm} \mathrm{Hg}$. The vitals shortly thereafter in the emergency department were heart rate of 93 beats per minute and blood pressure improved to $103 / 67 \mathrm{~mm} \mathrm{Hg}$. She was not icteric or pale and no cervical or axillary lymphadenopathy was appreciated. Lungs were clear to auscultation, with decreased breath sounds in the left lung base. No wheezes, rhonchi or crackles were appreciated. Cardiac examination was normal too. Abdominal examination was remarkable for a well-healed cesarean section scar. She had moderate tenderness in the left upper quadrant, with no rebound tenderness. No visceromegaly was appreciated. She had normal bowel sounds in all four quadrants. Rectal examination demonstrated a normal sphincteric tone and no external or internal hemorrhoids could be felt. Fecal occult test was negative. Neurological examination was unremarkable and extremity examination was within normal limits.

Patient was admitted for further management of her shortness of breath. Pulmonary embolism was high on the list of differential diagnosis. D-dimer levels were also elevated, prompting a computerized axial tomography with contrast (CAT) of the chest. CAT ruled out pulmonary embolism but did reveal severe circumferential wall thickening of the visualized splenic flexure highly suspicious for colitis or malignancy. It also revealed a $4 \mathrm{~mm}$ nodule in the left upper lobe of the lung. Dedicated CT imaging of the abdomen and pelvis with oral contrast was advised for further evaluation. CT of abdo- 


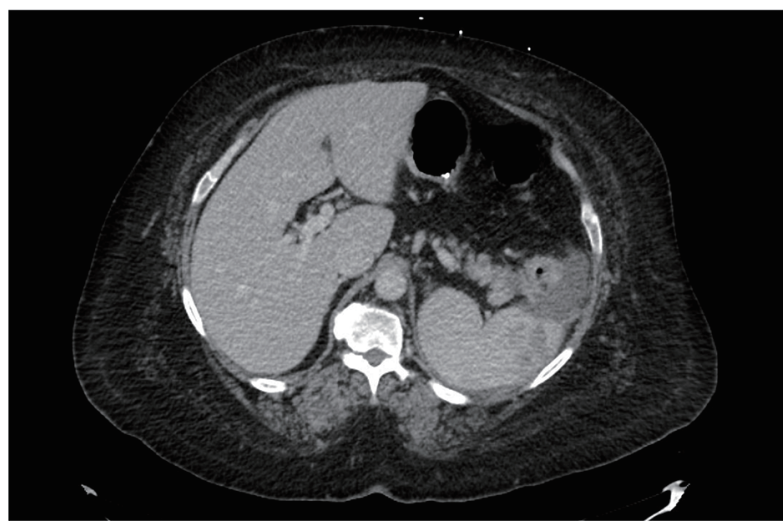

Figure 1. CT of abdomen showing a complex fluid regional to the spleen and mass-like focus extending from the spleen to the descending colon and the pancreatic tail.

men (Fig. 1) demonstrated complex fluid regional to the spleen and complex fluid or mass-like focus of decreased enhancement extending from the anterior lateral aspect of the spleen to the descending colon and to the pancreatic tail which could be inflammatory but was suspicious for malignancy. Fluid adjacent to the spleen was extending to the posterior cardia of the stomach. Irregular enhancement was noted in the lateral part of the spleen. Adjacent fat infiltration and enlarged regional lymph nodes to about $9 \mathrm{~mm}$ each were also noted.

Gastroenterology consult was requested and a colonoscopy was performed. Colonoscopy (Fig. 2) revealed a large tumor circumferential at the splenic flexure. It was friable and multiple biopsies were obtained from the tumor and the surrounding areas. Specimens were sent for pathological evaluation which revealed moderately differentiated invasive adenocarcinoma of the splenic flexure. Surgical intervention was planned and the patient underwent exploratory laparotomy followed by Hartmann's procedure (proctosigmoidectomy). Exploratory laparotomy revealed a tumor that was firmly adherent to the lateral wall of the colon and invading and extending into the spleen. The tumor appeared to be perforated but was contained within the abscess cavity and approximately $15 \mathrm{~mL}$ of pus was drained from the site. Liver appeared normal without any evidence of gross metastases. Patient tolerated the procedure well. After the surgery, she was assessed by oncology. Final pathology demonstrated a tumor, about $6 \mathrm{~cm}$ in greatest dimension in the splenic flexure with visible macroscopic perforation. Histologically it was a high grade poorly differentiated adenocarcinoma. Tumor has penetrated the surface of the visceral peritoneum. Vascular invasion was identified. The tumor was staged as stage III C tumor (T4b, N2a, M0). Also identified was a large area of acute inflammation, inflamed granulation tissue, abscess formation, vascular congestion, and hemorrhage associated with the area of tumor perforation at the splenic flexure region. Carcinoembryonic antigen which was ordered postoperatively was found to be elevated at 53.9 $\mathrm{ng} / \mathrm{mL}$ (normal is less than $4.8 \mathrm{ng} / \mathrm{mL}$ ). She was arranged for chemotherapy in an outpatient setting. Of note, during the hospital course, preoperatively the patient spiked a temperature of $100.4^{\circ} \mathrm{F}\left(38^{\circ} \mathrm{C}\right)$ and blood cultures sent during that time grew gram negative anaerobic bacilli in one anaerobic bottle, later identified as Bacteriodes species.
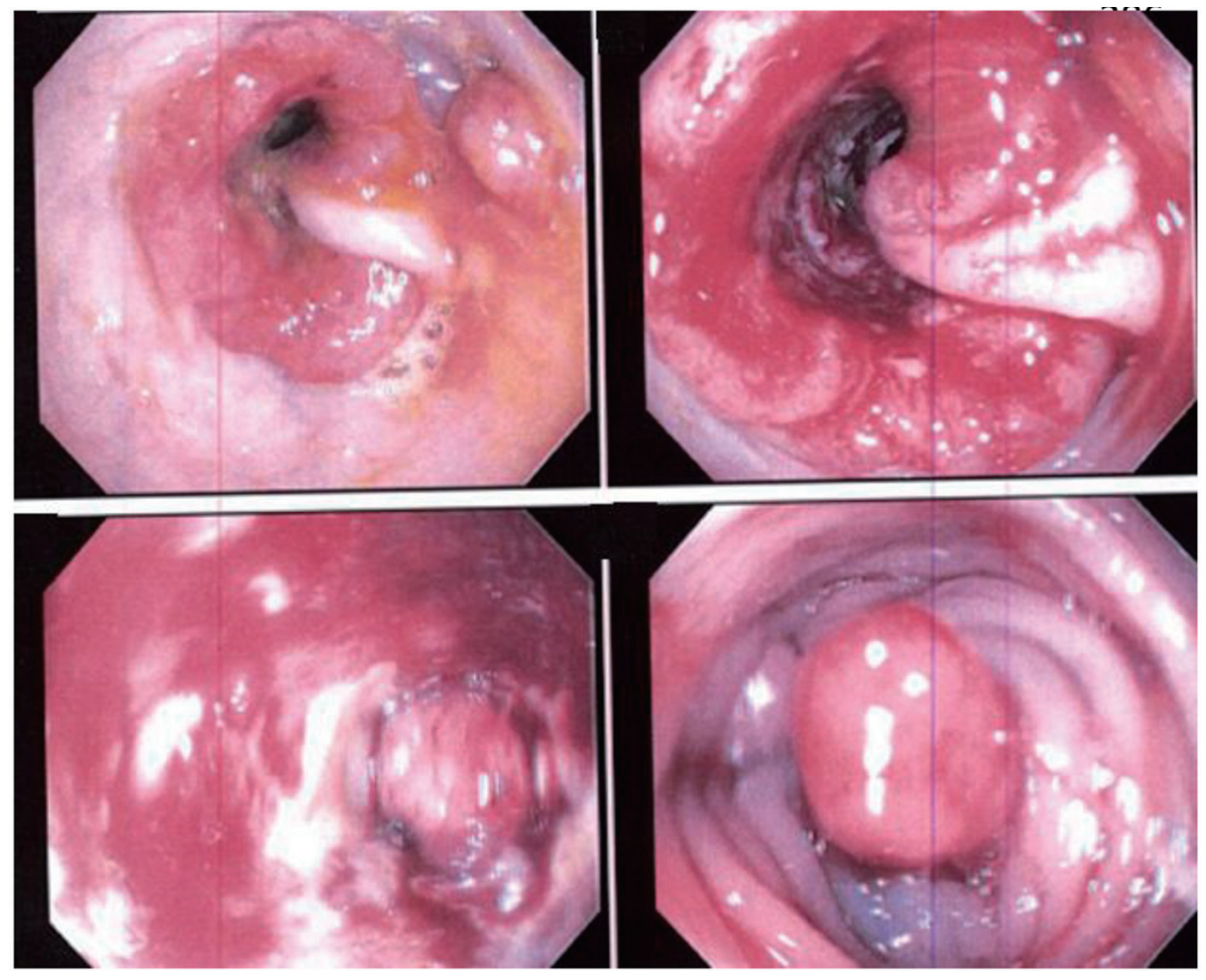

Figure 2. A large circumferential tumor at the splenic flexure. 


\section{Discussion}

One-third of the patients with colon cancer have major complications such as involvement of the adjacent organs or structures, obstruction, or perforation. Abscess formation, although rare, occurs in $0.3-4 \%$ of cases of colon cancer [1], and is the second most common presentation of perforation lesions. Perforation occurs in $2.5-10 \%$ of the patients with colon cancer [2, 3]. Michowitz et al have proposed the following clinical classification of perforation in regards to carcinoma of the colon [7]: 1) free perforation with leakage of the bowel contents into the peritoneal cavity; 2) covered perforation with local abscess formation; and 3) perforation into one of the neighboring organs or formation of a fistula.

Perforation in colon cancer occurs commonly, due either to direct perforation from the tumor necrosis or to blowout of proximal colon as a result of closed loop obstruction. Although the mechanism of abscess formation remains unclear, it is postulated that tumor growth may contribute to abscess formation in the presence of enteric organisms by outgrowing the blood supply. Suppuration due to leakage of bowel contents from the perforated tumors may lead to abscesses at various locations such as retroperitoneal, subcutaneous, peri-nephric, peri rectal and appendicular abscesses.

The clinic signs of a possible abscess formation include presence of fever and chills, abdominal pain varying in location and radiation depending upon the site of the abscess, and nausea and vomiting. Physical examination findings mainly depend on the location of the abscess and may include a palpable tender mass, splenomegaly, muscle guarding, costovertebral angle tenderness, increased or absent bowel sounds, and shallow breathing due to diaphragmatic splinting. The accurate preoperative diagnosis of abscess formation related to colon cancer is considered difficult. Supportive laboratory findings include leukocytosis with a left shift and microcytic anemia due to the chronic blood loss from the tumor. Basic metabolic panel may demonstrate signs of dehydration with elevated blood urea nitrogen and creatinine levels and increased lactate depending on the severity of the sepsis. Heme-occult testing may be positive or negative. Blood cultures usually grow enteric gram-negative and anaerobic organisms (Streptococcus bovis and Bacteriodes fragilis). Carcinoembryonic antigen levels are usually elevated but since they are neither sensitive nor specific, they are not useful in the diagnosis of colon cancer or its complications.

Conventional radiographic studies such as X-rays detect indirect signs such as displacement of the ureters, alteration of the psoas margin, ileus, abnormal masses and extra luminal gas, elevation of the hemi-diaphragm, and atelectasis with pleural effusions. Barium enema, though not indicated in every case, may demonstrate a neoplasm, a fistulous tract, or an abscess cavity. More advanced imaging like a CAT scan in most cases reveals relatively severe pericolic fat stranding adjacent to a thickened colon wall [4]. However, if perforation or abscess is present in patients with colon cancer, then physicians can be faced with a diagnostic uncertainty. Evaluation and characterization of a thickened bowel wall is needed in this situation. A marked asymmetric wall thickening with loss of stratified enhancement that involves a short segment of the colon favors a diagnosis of an underlying malignancy, while preserved layering of a thickened bowel wall is an important feature favoring the diagnosis of benign inflammatory conditions $[5,6]$. Moreover, an abrupt transition from a normal to an abnormal bowel wall is characteristic of malignancy, whereas a smooth, gradual, long transition is usually present in benign conditions [5]. The presence of pericolic lymphadenopathy is more commonly found in colon cancer than it is in benign conditions $[5,6]$. CAT scan is useful for providing valuable staging information for the preoperative assessment. Colonoscopy is most useful for direct objective investigation of the tumor features, including taking biopsies and planning further intervention. Exploratory laparotomy is an important modality both in diagnosis and the treatment as it can expose an abscess which imaging studies cannot reveal at times.

Patients with colorectal cancer who present with major complications are generally considered to have lower crude survival and higher operative mortality rates. Michowitz et al [7] have reported operative mortality and 5-year survival rates of $50 \%$ and $20 \%$, respectively. No large studies have evaluated operative mortality, morbidity, and prognosis in such patients. Complete resection of colon cancer and the abscess wall is preferable, but the patients' co-morbidities and procedural tolerability come into play before pursuing a major surgical decision. If the surgery is contraindicated in light of patient's medical condition, percutaneous aspiration and indwelling catheter drainage combined with antibiotic therapy may be immediately started without further diagnostic workup. In patients with low surgical risk, immediate exploratory laparotomy can be performed with drainage of the abscess, colectomy, and diverting colostomy. It should be noted that the survival time is not influenced by the type of perforation but is influenced by the stage of tumor invasion

\section{Grant Support}

None.

\section{Financial Disclosures}

None.

\section{Conflicts of Interest}

None.

\section{References}

1. Okita A, Kubo Y, Tanada M, Kurita A, Takashima S. Unusual abscesses associated with colon cancer: report of three cases. Acta Med Okayama. 2007;61(2):107-113.

2. Hulnick DH, Megibow AJ, Balthazar EJ, Gordon RB, Surapenini R, Bosniak MA. Perforated colorectal neo- 
plasms: correlation of clinical, contrast enema, and CT examinations. Radiology. 1987;164(3):611-615.

3. Tsai HL, Hsieh JS, Yu FJ, Wu DC, Chen FM, Huang CJ, Huang YS, et al. Perforated colonic cancer presenting as intra-abdominal abscess. Int $\mathrm{J}$ Colorectal Dis. 2007;22(1):15-19.

4. Filippone A, Ambrosini R, Fuschi M, Marinelli T, Genovesi D, Bonomo L. Preoperative T and N staging of colorectal cancer: accuracy of contrast-enhanced multidetector row CT colonography - initial experience. Radiology. 2004;231(1):83-90.
5. Hoeffel C, Crema MD, Belkacem A, Azizi L, Lewin M, Arrive L, Tubiana JM. Multi-detector row CT: spectrum of diseases involving the ileocecal area. Radiographics. 2006;26(5):1373-1390.

6. Pereira JM, Sirlin CB, Pinto PS, Jeffrey RB, Stella DL, Casola G. Disproportionate fat stranding: a helpful CT sign in patients with acute abdominal pain. Radiographics. 2004;24(3):703-715.

7. Michowitz M, Avnieli D, Lazarovici I, Solowiejczyk M. Perforation complicating carcinoma of colon. J Surg Oncol. 1982;19(1):18-21. 